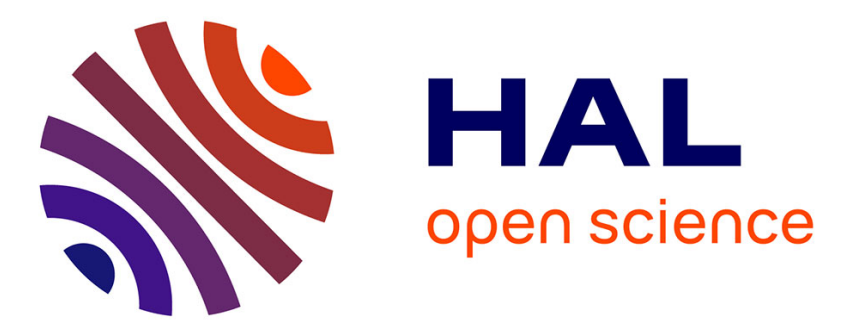

\title{
Novel molecule combinations and corresponding hybrids targeting artemisinin-resistant Plasmodium falciparum parasites
}

Manel Ouji, Michel Nguyen, Romain Mustière, Tony Jimenez, Jean-Michel Augereau, Françoise Benoit-Vical, Céline Deraeve

\section{To cite this version:}

Manel Ouji, Michel Nguyen, Romain Mustière, Tony Jimenez, Jean-Michel Augereau, et al.. Novel molecule combinations and corresponding hybrids targeting artemisinin-resistant Plasmodium falciparum parasites. Bioorganic \& Medicinal Chemistry Letters, 2021, 39, pp.127884. 10.1016/j.bmcl.2021.127884 . hal-03170976

\section{HAL Id: hal-03170976 https://hal.science/hal-03170976}

Submitted on 16 Mar 2021

HAL is a multi-disciplinary open access archive for the deposit and dissemination of scientific research documents, whether they are published or not. The documents may come from teaching and research institutions in France or abroad, or from public or private research centers.
L'archive ouverte pluridisciplinaire HAL, est destinée au dépôt et à la diffusion de documents scientifiques de niveau recherche, publiés ou non, émanant des établissements d'enseignement et de recherche français ou étrangers, des laboratoires publics ou privés. 


\title{
Novel molecule combinations and corresponding hybrids targeting artemisinin-resistant Plasmodium falciparum parasites.
}

\author{
Manel Ouji ${ }^{\mathrm{a}, \mathrm{b}, \mathrm{c}}$, Michel Nguyen ${ }^{\mathrm{a}, \mathrm{b}, \mathrm{c}}$, Romain Mustière ${ }^{\mathrm{a}}$, Tony Jimenez ${ }^{\mathrm{a}}$, \\ Jean-Michel Augereau ${ }^{\mathrm{a}, \mathrm{b}, \mathrm{c}}$, Françoise Benoit-Vical ${ }^{\mathrm{a}, \mathrm{b}, \mathrm{c},{ }^{*}}$, and Céline Deraeve ${ }^{\mathrm{a},{ }^{*}}$ \\ ${ }^{\text {a }}$ LCC-CNRS, Laboratoire de Chimie de Coordination, Université de Toulouse, CNRS, UPS, Toulouse, \\ France \\ ${ }^{\mathrm{b}}$ Institut de Pharmacologie et de Biologie Structurale, IPBS, Université de Toulouse, CNRS, UPS, \\ France \\ ${ }^{c}$ New antimalarial molecules and pharmacological approaches, ERL Inserm UMR 1289, Toulouse, \\ France \\ * Corresponding authors: FBV (francoise.vical@inserm.fr); CD (celine.deraeve@ lcc-toulouse.fr).
}

\begin{abstract}
Malaria is still considered as the major parasitic disease and the development of artemisinin resistance does not improve this alarming situation. Based on the recent identification of relevant malaria targets in the artemisinin resistance context, novel drug combinations were evaluated against artemisinin-sensitive and artemisinin-resistant Plasmodium falciparum parasites. Corresponding hybrid molecules were also synthesized and evaluated for comparison with combinations and individual pharmacophores (e.g. atovaquone, mefloquine or triclosan). Combinations and hybrids showed remarkable antimalarial activity $\left(\mathrm{IC}_{50}=0.6\right.$ to $1.1 \mathrm{nM}$ for the best compounds), strong selectivity, and didn't present any cross-resistance with artemisinin. Moreover, the combination triclosan + atovaquone showed high activity against artemisinin-resistant parasites at the quiescent stage but the corresponding hybrid lost this pharmacological property. This result is essential since only few molecules active against quiescent artemisinin-resistant parasites are reported. Our promising results highlight the potential of these combinations and paves the way for pharmacomodulation work on the best hybrids.
\end{abstract}

Keywords: Antimalarial drug - Plasmodium falciparum - Artemisinin resistance - Quiescence

- Drug combination - Hybrid molecule 
Malaria is one of the leading causes of mortality by infectious diseases, killing more than 400000 people each year, mainly in tropical and subtropical regions. ${ }^{1}$ The disease is caused by the Plasmodium parasite, transmitted through the bite of an infected mosquito of the genus Anopheles. A notable decrease of malaria mortality has been observed for the 20 last years due in part to the introduction of artemisinin and its derivatives (ARTs) in the antimalarial therapeutic arsenal. To reduce the risk of drug resistance, ARTs are used in combination with one or two other antimalarial drugs having different modes of action and pharmacokinetic properties. These Artemisinin-based Combination Therapies (ACTs) have been recommended by the WHO, since 2001, as first-line treatments of uncomplicated falciparum malaria worldwide. ${ }^{2}$ However, the efficacy of the ACTs rapidly declined in South-East Asia, due to the emergence of $P$. falciparum resistance to ARTs ${ }^{3-7}$ but also to partner drugs, ${ }^{8,9}$ and is now threatening malaria eradication. Targeting ARTs-resistant parasites is thus an urgent concern.

ART-resistance is based on an original mechanism relying on a parasite quiescence state induced by ARTs exposure. ${ }^{10-12}$ Indeed, this state of quiescence is characterized by a drastically lowered metabolism that allows parasites to limit ART-induced cellular damages. ${ }^{13,14}$ However, quiescent parasites still possess a maintained mitochondrial activity and an implemented fatty acid synthesis type II (FAS-II) pathway in the apicoplast that enable parasites to restart their cell cycle after drug elimination. ${ }^{10,13,14}$ Interestingly, the antimalarial drug atovaquone (ATQ, Table 1), which targets the $\mathrm{bc}_{1}$ complex of mitochondrial electron transport chain, ${ }^{15}$ was reported to kill dihydroartemisinin-induced quiescent parasites. ${ }^{16,17}$ Similarly, triclosan (TCS) and haloxyfop, which inhibit the FabI and acetyl-CoA carboxylase of the Plasmodium FAS-II pathway respectively, delayed the recrudescence of quiescent parasites after dihydroartemisinin treatment. ${ }^{13}$ Simultaneously targeting the mitochondrion with ATQ and the apicoplast with TCS would thus be of valuable interest to face the issue of ART-resistance. Compound GW844520 reported, like ATQ, as an inhibitor of the bc1 complex of the mitochondrial electron transport chain, ${ }^{18}$ was also selected to be studied in the context of ART-resistance. Finally, the antimalarial drug mefloquine (MQ) was picked out as a relevant compound for drug combinations studies because it is one of the only two partner drugs used in ACTs to be reported as active on quiescent ART-resistant parasites. ${ }^{[17]}$ We report here the evaluation of different combinations of two of these compounds, each targeting one essential pathway for quiescence survival: ATQ + TCS, ATQ + MQ, GW844520 + TCS. This evaluation was performed first on proliferating parasites then in an artemisinin resistance context on dihydroartemisinin (DHA)induced quiescent state, thanks to two specific tests, the recrudescence assay, ${ }^{19}$ and the 
Quiescent-stage Survival Assay (QSA) for the best combination. ${ }^{17}$ In addition, the very high antiplasmodial activities reported with these combinations lead us to a hybridization strategy. Hybrid molecules, combining at least two pharmacophoric subunits with distinct modes of action, are considered as original compounds which offer the potential of improved biological activity, reduced risk of drug resistance emergence and better patient compliance. Compared to simple drug combinations, they also offer easier formulation, as well as more predictable pharmacokinetic and pharmacodynamic relationships. ${ }^{20,21}$ Several reviews dealing with recent development of antimalarial hybrids are available in the literature. ${ }^{22,23}$ Combinations of selected compounds that showed activity both on proliferating parasites and on quiescent parasites were thus translated to corresponding hybrid molecules: ATQ-TCS, ATQ-MQ and GW844520TCS. The synthesis of these original compounds is reported herein, together with their evaluation for in vitro antiparasitic activity against ART-susceptible and ART-resistant strains of P. falciparum (chemosensitivity, recrudescence assay, and QSA for the best hybrid).

Table 1. Structures and targets of compounds reported as active on ART- or DHAinduced quiescent parasites (ATQ, TCS, haloxyfop) ${ }^{13,16}$ or which have showed activity on ARTs-resistant parasites maintained in a quiescent state a (ATQ, MQ, GW844520). ${ }^{17}$

Main targets (P. falciparum)
Ref

a Activity measured by Quiescent-stage Survival Assay (QSA) 
Drug combinations targeting different pathways were first evaluated for antiplasmodial effect on proliferating P. falciparum. With an $\mathrm{IC}_{50}$ value of $2 \mathrm{nM}$, atovaquone (ATQ) displayed a very high activity on proliferating parasites. GW844520 and mefloquine (MQ) also confirmed their antiplasmodial activity with $\mathrm{IC}_{50}$ values in the $40-90 \mathrm{nM}$ range (Table 2). Significantly less active, triclosan had a low antiplasmodial activity with an $\mathrm{IC}_{50}$ of $5 \mu \mathrm{M}$. Interestingly the 1:1 combination ATQ + MQ supports the strategy to target different parasite pathways, with an $\mathrm{IC}_{50}$ value of $0.9 \mathrm{nM}$, corresponding to a more potent anti-proliferative effect than the two compounds alone. This combination appeared as the best one tested. The combination ATQ + TCS also had a good antiplasmodial activity but its $\mathrm{IC}_{50}$ value $(1.8 \mathrm{nM})$ close to the ATQ one suggests that the result is mainly due to ATQ activity. The same reasoning can be made for the combination GW844520 + TCS. The low efficacy of TCS, alone and in combination, can be explained by the fact that it targets lipid metabolism, while this metabolism is absent in proliferating parasites. By contrast, it has been shown that parasite lipid metabolism takes place during induced quiescence of the parasites by dihydroartemisinin treatment. ${ }^{13}$ In these conditions, TCS would inhibit the FabI enzyme of the Plasmodium FAS-II pathway and delay the recrudescence of quiescent parasites. ${ }^{13}$ Moreover, good selectivity of these molecules towards mammalian cells (Table 2) led us to pursue their evaluation in an artemisinin resistance context.

Table 2: Antimalarial and cytotoxic activities of selected molecules and their (1:1) combinations.

\begin{tabular}{|c|c|c|c|c|}
\hline Compound & $\begin{array}{c}\text { Hybrid } \\
\text { pharmacophoric } \\
\text { units }\end{array}$ & $\begin{array}{c}\text { Antiplasmodial } \\
\text { activity on } \\
\text { P. falciparum } \\
\text { IC }_{50} \pm \text { SEM (nM) }\end{array}$ & $\begin{array}{c}\text { Cytotoxicity } \\
\text { on Vero Cells } \\
\text { CC }_{50} \pm \text { SEM }(\mu M)\end{array}$ & $\begin{array}{l}\text { Selectivity index } \\
\text { CC }_{50} \text { Vero Cells / } \\
\text { IC }_{50} P \text {. falciparum }\end{array}$ \\
\hline ATQ & - & $2.1 \pm 0.6$ & $0.5 \pm 0.1$ & 257 \\
\hline GW844520 & - & $42 \pm 10.5$ & $8 \pm 1$ & 190 \\
\hline MQ & - & $87 \pm 18$ & $21 \pm 7$ & 241 \\
\hline TCS & - & $510^{3} \pm 110^{3}$ & $25 \pm 9$ & 5 \\
\hline $\mathrm{ATQ}+\mathrm{TCS}$ & - & $1.8 \pm 0.8$ & - & \\
\hline
\end{tabular}




\begin{tabular}{ccccc}
\hline GW844520 + TCS & - & $38 \pm 6$ & - & \\
\hline ATQ + MQ & - & $0.9 \pm 0.3$ & $6 \pm 2$ & 10000 \\
\hline Hybrid 4 & ATQ / TCS & $0.6 \pm 0.5$ & $>120$ & $>710$ \\
\hline Hybrid 8 & GW844520 / TCS & $169 \pm 37$ & $4 \pm 1$ & 6660 \\
\hline Hybrid 12 & ATQ / MQ & $0.6 \pm 0.3$ & $5 \pm 2$ & 4545 \\
\hline Hybrid 13 & ATQ / MQ & $1.1 \pm 1.0$ & 130 & 7222 \\
\hline artemisinin & - & $18 \pm 2.5$ & \\
\hline
\end{tabular}

$\mathrm{IC}_{50}$ values on Plasmodium falciparum F32-TEM strain were obtained using SYBR Green assay. Cytotoxicity activities were evaluated against Vero cell line. Artemisinin was used as antiplasmodial control drug.

ATQ: atovaquone; MQ: mefloquine; TCS: triclosan.

Number of different independent experiments performed: $\mathrm{n}=4$ for the cytotoxicity assay; at least $\mathrm{n}=4$ for the antiplasmodial activity. For each one, a triplicate (technical repeats) was carried out.

The Plasmodium artemisinin resistance mechanism consists in a quiescence phenomenon based on a cell cycle arrest of a sub-population of the parasites during artemisinin or its derivatives treatment. When the drug is eliminated, the parasites are able to develop again normally. ${ }^{10,32}$ This quiescence is characterized by a DNA and RNA synthesis arrest under treatment. ${ }^{13} \mathrm{~A}$ standard chemosensitivity assay, based on the measurement of the inhibition of parasite proliferation, is thus not relevant for the evaluation of compounds against artemisinin-resistant parasites. Indeed, there is no difference in $\mathrm{IC}_{50}$ values between artemisinin-resistant and artemisinin-susceptible strains. ${ }^{10,19}$ In this context, the recrudescence assay, based on the comparison of recrudescence capacities between the artemisinin-resistant strain F32-ART5 and its twin artemisinin-sensitive strain F32-TEM after $48 \mathrm{~h}$ of exposure with the molecule of interest, was used to determine if a cross-resistance exists with artemisinin. Potential crossresistance is evidenced by a faster resumption of the ART-resistant strain (F32-ART5) compared to the sensitive F32-TEM parasites. Interestingly, no significant differences of recrudescence were observed between F32-ART5 and F32-TEM neither for the compounds tested alone nor for the three combinations (Table 3), thus demonstrating the absence of crossresistance with artemisinin.

Table 3: Recrudescence capacity of Plasmodium falciparum F32-ART5 and F32-TEM parasites after $48 \mathrm{~h}$ of drug exposure. 


\begin{tabular}{|c|c|c|c|c|c|}
\hline \multirow{3}{*}{ Compounds } & \multirow{3}{*}{ Doses } & \multicolumn{2}{|c|}{ Median recrudescence days } & \multirow{3}{*}{$\begin{array}{c}\text { Delay in } \\
\text { recrudescence }^{\text {time }} \\
\text { Mean }^{\text {a }} \\
\text { SEM }\end{array}$} & \multirow{3}{*}{$P$-value ${ }^{b}$} \\
\hline & & F32-ART5 & F32-TEM & & \\
\hline & & & & & \\
\hline \multirow[t]{2}{*}{ ATQ } & $1 \mu \mathrm{M}$ & 11 & 14 & 3 & - \\
\hline & $7 \mu \mathrm{M}$ & 21 & 23 & $1 \pm 0.6$ & 0.486 \\
\hline GW844520 & $7 \mu \mathrm{M}$ & 2 & 8 & $3 \pm 1.2$ & 0.325 \\
\hline MQ & $1 \mu \mathrm{M}$ & $>30$ & $>30$ & - & - \\
\hline TCS & $7 \mu \mathrm{M}$ & 5 & 6 & $0.8 \pm 0.9$ & 0.417 \\
\hline ATQ + TCS $(1: 1)$ & $7 \mu \mathrm{M}$ & 17 & 19.5 & $2.3 \pm 1.1$ & 0.175 \\
\hline GW844520 + TCS (1:1) & $7 \mu \mathrm{M}$ & 8 & 12 & $3.3 \pm 1.4$ & 0.418 \\
\hline ATQ + MQ (1:1) & $1 \mu \mathrm{M}$ & $>30$ & $>30$ & - & - \\
\hline Hybrid 4 (ATQ/TCS) & $7 \mu \mathrm{M}$ & 9 & 12 & $4 \pm 2.4$ & 0.265 \\
\hline Hybrid $\mathbf{8}$ & $7 \mu \mathrm{M}$ & 3 & 4 & $0.6 \pm 0.4$ & 0.093 \\
\hline (GW844520/TCS) & $20 \mu \mathrm{M}$ & 2 & 3 & 1 & - \\
\hline Hybrid 12 (ATQ/MQ) & $1 \mu \mathrm{M}$ & 14 & 14 & 0 & - \\
\hline Hybrid 13 (ATQ/MQ) & $1 \mu \mathrm{M}$ & 14 & 14 & 0 & - \\
\hline Artemisinin $^{c}$ & $18 \mu \mathrm{M}$ & 9 & 22 & $12.5 \pm 1.8$ & $<0.0001$ \\
\hline
\end{tabular}

Each experiment was performed for F32-ART5 and F32-TEM cultivated in parallel in the same conditions (adjusted to the same initial parasitemia and cultivated with the same batch of erythrocytes and same batch of human serum) to generate paired results.

Synchronized ring-stage parasites have undergone $48 \mathrm{~h}$ of drug treatment. After culture washing, the parasitemia was monitored during 30 days or until reaching the initial parasitemia, defined as the recrudescence day. If no parasites were observed at the end of the experiment, the culture was considered as no recrudescent, and the recrudescence day was noted as $>30$. The doses tested for ATQ correspond to concentrations pharmacologically relevant in patients. The same doses were then used for all the compounds tested. The results correspond to data obtained in at least 3 independent experiments except for the molecules 12, 13, ATQ $(1 \mu \mathrm{M})$, MQ and ATQ + MQ (1:1), 1 time. The same number of experiments was performed for each parasite lineage and statistically analyzed.

${ }^{\mathrm{a}}$ The delay of recrudescence days corresponds to the mean of the differences, obtained for each experiment, between the day of recrudescence of F32-ART5 compared to F32-TEM, after $48 \mathrm{~h}$ of molecule exposure.

b A log-rank (Mantel-Cox) test was used for statistical analysis of recrudescence days; a significant difference between F32-ART5 and F32-TEM is validated for $\mathrm{p}<0.05$.

c The concentration of artemisinin at $18 \mu \mathrm{M}$ was shown as the most relevant to discriminate both strains F32-

ART5 and F32-TEM regarding artemisinin sensitivity. ${ }^{19}$

ATQ: atovaquone; MQ: mefloquine; TCS: triclosan. 
The good $\mathrm{IC}_{50}$ values for the three combinations, associated with good selectivity indexes and absence of cross-resistance with artemisinin, motivated the design of hybrid compounds inspired from such combinations. The first hybrid was thus based on the association of ATQ and TCS bound via an 8-carbon chain, in order to limit steric hindrance. Since ester derivatives of ATQ and TCS have shown antimalarial activities comparable to the one of their parent drugs,${ }^{33,34}$ the free -OH groups of both drugs were selected for linker attachment through ester bonds from octanedioic acid. Ethers or carbamate bonds have also been considered for linker attachment to the -OH groups. However, El Hage et al. reported that ester analogues of atovaquone were more active than the corresponding ethers ${ }^{33}$ and carbamate derivatives of atovaquone were not selected because such compounds are reported as chemically unstable. ${ }^{35,36}$ Hybrid 4 was thus prepared in a four steps synthesis (Scheme 1). Octanedioic acid was monoprotected via a Steglich esterification reaction performed with benzylic alcohol, in the presence of dicyclohexylcarbodiimide (DCC) and a catalytic amount of 3,4dimethylaminopyridine (DMAP), with a yield of $71 \%$. The remaining carboxylic acid function of 1 was then activated in the presence of thionyl chloride and reacted with TCS, to afford the intermediate 2 in $61 \%$ yield. Finally, quantitative deprotection of the benzylic ester by catalytic hydrogenation on $\mathrm{Pd} / \mathrm{C}$, activation of the resulting carboxylic acid with thionyl chloride and subsequent esterification with ATQ afforded hybrid 4, which was isolated in $89 \%$ yield.

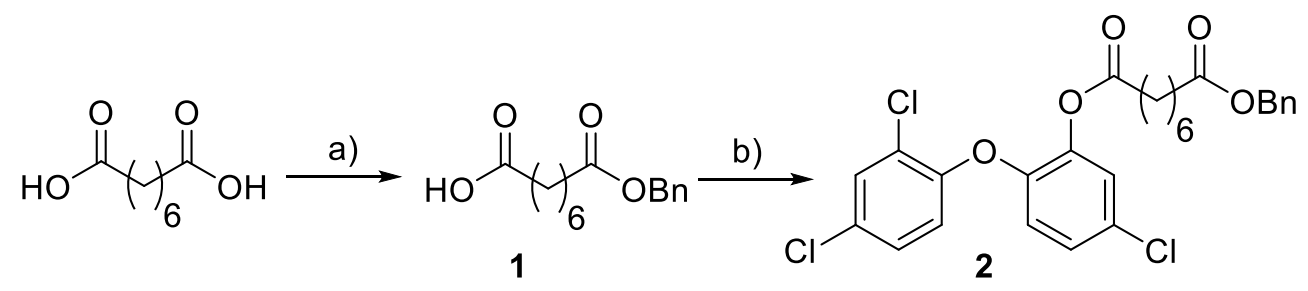<smiles>CC(C)(C)C(=O)OC1=C([C@H]2CC[C@H](c3ccc(Cl)cc3)CC2)C(=O)c2ccccc2C1=O</smiles>

Scheme 1. Synthesis of hybrid 4. Reagents and reaction conditions: (a). Benzyl alcohol, DMAP, DCC, DCM, rt, $1 \mathrm{~h}, 71 \%$; (b). i) $\mathrm{SOCl}_{2}, 50{ }^{\circ} \mathrm{C}, 3 \mathrm{~h}$, ii) TCS, pyridine, DCM, $0{ }^{\circ} \mathrm{C}$ to rt, 8 h, $61 \%$; (c). $\mathrm{H}_{2}, \mathrm{Pd} / \mathrm{C}, \mathrm{EtOAc}, \mathrm{rt}$, overnight, $100 \%$; (d). i) $\mathrm{SOCl}_{2}, 50{ }^{\circ} \mathrm{C}, 3 \mathrm{~h}$, ii), ATQ, pyridine, DCM, rt, overnight, $89 \%$. 
A second hybrid, for which the ATQ moiety of 4 was replaced by the GW844520 unit, was prepared according to a similar sequence (Scheme 2). GW844520 (7) ${ }^{37}$ was first prepared via a Suzuki-Miyaura cross-coupling reaction between 3-chloro-5-iodo-2,6-dimethylpyridin4(1H)-one $5^{38}$ and boronic acid $6,{ }^{37}$ under microwave conditions. Compound 7 was then treated with sodium hydride and the resulting pyridinolate intermediate was reacted with the acyl chloride of compound $\mathbf{3}$, to give hybrid $\mathbf{8}$ with a yield of $30 \%$.

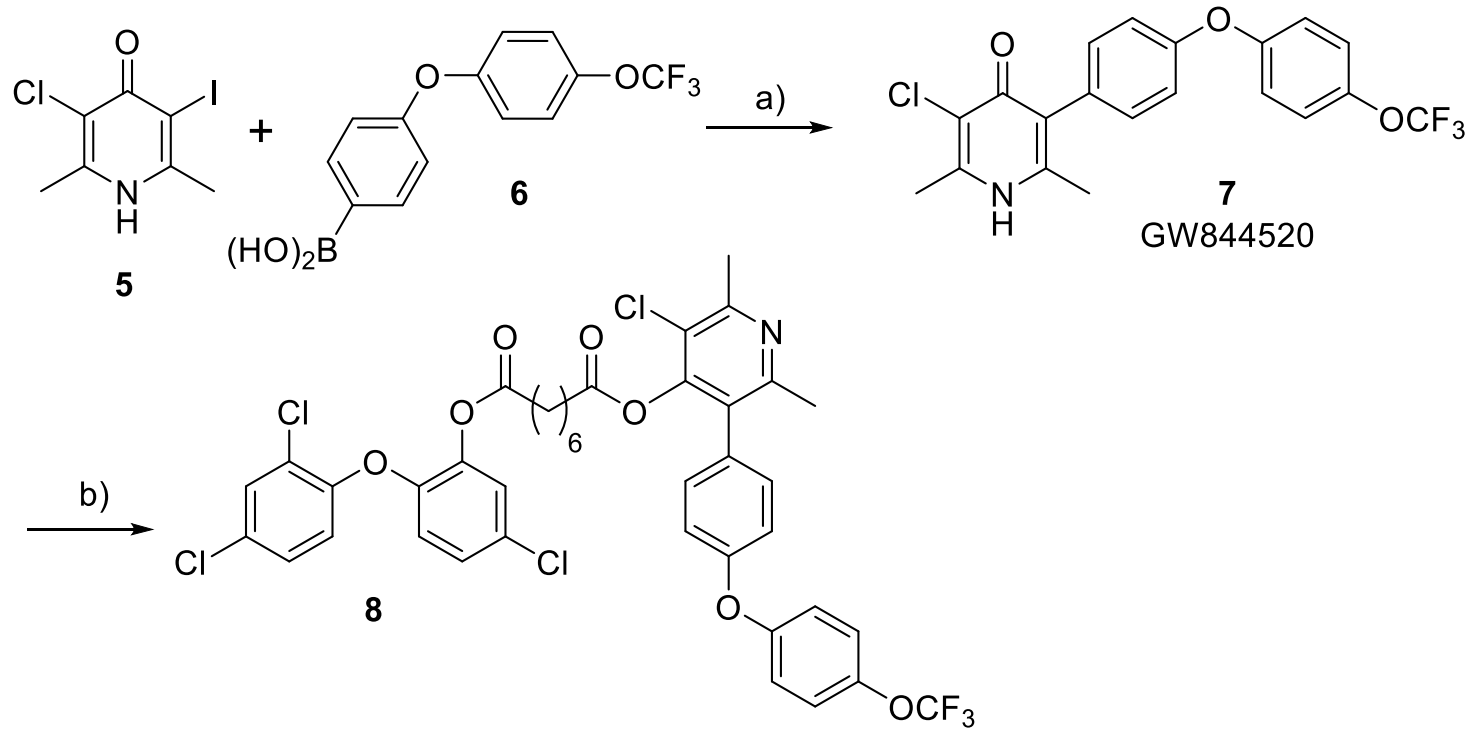

Scheme 2. Synthesis of hybrid 8. Reagents and reaction conditions: (a). $\mathrm{Pd}(\mathrm{OAc})_{2}, \mathrm{~K}_{2} \mathrm{CO}_{3}, n-\mathrm{Bu} \mathrm{HBr}_{4}, \mathrm{EtOH}$, $100{ }^{\circ} \mathrm{C}, 1 \mathrm{~h}, \mathrm{MW}, 29 \%$; (b). i) $\mathrm{NaH}$, THF, rt, $10 \mathrm{~min}$, ii) $\left(3+\mathrm{SOCl}_{2}\right), \mathrm{THF}, \mathrm{rt}$, overnight, $30 \%$.

The two last hybrids $\mathbf{1 2}$ and $\mathbf{1 3}$ were designed by associating the two antimalarial drugs ATQ and MQ, bound via a diester linker, as for the ATQ-containing hybrid 4. The selection of an ester bond for MQ linking allowed the direct transposition of the previous synthesis scheme using a symmetrical linker, and was supported by the antiplasmodial and antimalarial activity of a trifluoromethylartemisinin-mefloquine hybrid displaying similar linker attachment. ${ }^{39}$ Our first attempts to use octanedioic acid as linker precursor, as for hybrids $\mathbf{4}$ and $\mathbf{8}$, having been unsuccessful, we envisaged shorter linkers accessible from sucininc and glutaric anhydrides. The synthesis pathway for hybrids $\mathbf{1 2}$ and $\mathbf{1 3}$ is presented in Scheme 3. The first step consisted in the protection of the piperidinyl amine of MQ by a Boc group, followed by treatment of the resulting Boc-mefloquine 9 with succinic or glutaric anhydride to give compounds $\mathbf{1 0}{ }^{39}$ and 11, with overall yields of $94 \%$ and $92 \%$. The free carboxylic acids of these two compounds were then esterified by ATQ after an activation step with thionyl chloride. During the course of 
the reaction, the piperidinyl amine was (partially) deprotected, affording hybrids $\mathbf{1 2}$ (and 12Boc) and 13, respectively. 12-Boc, isolated with a yield of $21 \%$, was finally converted to hybrid 12 by treatment with $\mathrm{HCl}$.

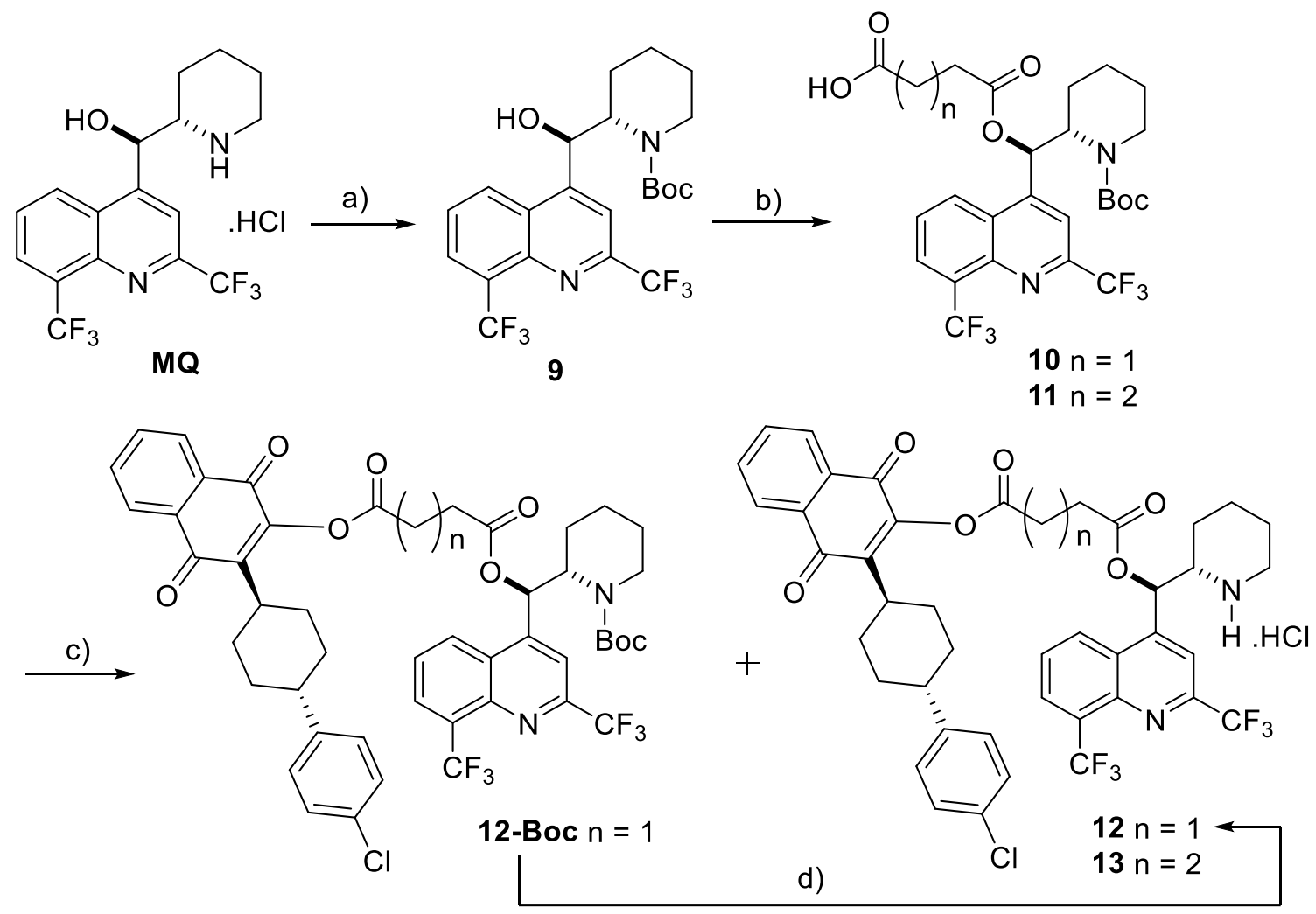

Scheme 3. Synthesis of hybrids 12 and 13. Reagents and reaction conditions: (a). $\mathrm{Boc}_{2} \mathrm{O}, \mathrm{Et}_{3} \mathrm{~N}, \mathrm{DCM}, 95 \%$; (b) succinic anhydride or glutaric anhydride, $\mathrm{Et}_{3} \mathrm{~N}, \mathrm{CHCl}_{3}, 99 \%$ and $97 \%$, respectively; (c). i) $\mathrm{SOCl}_{2}$, ii) ATQ, pyridine, DCM, $11 \%$ for $\mathbf{1 2}$ (+ $21 \%$ 12-Boc), $27 \%$ for 13; (d). HCl, MeOH, $96 \%$.

Hybrids were first evaluated for their activity on proliferating parasites. Hybrid 4 (ATQ/TCS) showed a sub-nanomolar $\mathrm{IC}_{50}$ value $(0.6 \mathrm{nM})$ against $P$. falciparum, better than the corresponding pharmacophores alone and their combination (Table 2). Similarly, hybrids 12 and 13 (ATQ/MQ) displayed $\mathrm{IC}_{50}$ values of 0.6 and $1.1 \mathrm{nM}$, respectively, comparable to the one of the ATQ + MQ combination and better than the activities of the individual drugs. By contrast hybrid 8, resulting from the association of GW844520 and TCS, was significantly less active than the corresponding combination and GW844520 alone (Table 2). One explanation for this reduced activity could be a default of aqueous solubility of the hybrid, lower than the one of GW844520, which was already described as poorly soluble. ${ }^{40}$ Otherwise, the linker attachment in hybrid 8 compared to GW844520 may affect target interaction. Indeed, the modification introduced in hybrid $\mathbf{8}$ locked the 4-pyridone moiety in the less favored pyridinol 
tautomeric form, ${ }^{37}$ preventing H-bond formation between the pyridine carbonyl group and Ser35 residue of cytochrome $b_{c}{ }_{1}{ }^{28}$

Interestingly, the cytotoxicity of the four hybrids was very low (Table 2), resulting in excellent selectivity indexes.

The recrudescence assay (Table 3) showed a loss of activity of all hybrids compared to their corresponding combinations with more days necessary for parasites to reach initial parasitemia when they are treated by combinations than for parasites treated by the corresponding hybrids. Surprisingly, this activity decrease of hybrids is only noted when high concentrations are tested (recrudescence assay, Table 3) but not for lower ones ( $\mathrm{IC}_{50}$ values, Table 2). This result could indicate that the hybrids are less soluble than the corresponding combinations. Interestingly, for all the hybrid compounds, no cross-resistance with artemisinin was reported since no significant difference in recrudescence capacity of the parasites was observed between the two strains, like for the compounds alone and their combinations. The difference of 12.5 days between the two strains, F32-ART5 and F32-TEM, can be noted for the control drug artemisinin as the sign of the resistance of the strain F32-ART5 (Table 3).

As the mitochondrial electron transfer chain and the apicoplast FAS-II pathway are described as maintained active during dihydroartemisinin-induced quiescence, ${ }^{13}$ and because of their very high antiplasmodial activity reported, the ATQ + TCS combination $\left(\mathrm{IC}_{50}=1.8 \mathrm{nM}\right)$ and its corresponding hybrid molecule $4\left(\mathrm{IC}_{50}=0.6 \mathrm{nM}\right)$ were selected for evaluation on artemisininresistant quiescent parasites, using the Quiescent-stage Survival Assay (QSA) (Table 4). The parent molecules (ATQ and TCS) were also evaluated for comparison. In the QSA, quiescence is first induced with 6 h-DHA treatment then parasites are exposed to the drug to be tested for $48 \mathrm{~h}$ in the presence of DHA to maintain the quiescence state. QSA interpretation is based on the difference in recrudescence days after exposure of DHA-induced quiescent parasites to the compound being tested (DHA $6 \mathrm{~h} /(\mathrm{DHA}+$ molecule) $48 \mathrm{~h}$ ) compared to DHA alone treatment (DHA $6 \mathrm{~h} /$ DHA $48 \mathrm{~h}$ ). ${ }^{17}$ A cut-off of 6 days-delay is reported as significant. ${ }^{17}$ The control drug chloroquine shows that a molecule can be active on proliferative parasites (Table 4 , third column) but lose its activity on quiescent parasites, with no significant difference between the two first columns. The delay of 13 days in the recrudescence time observed between DHA $6 \mathrm{~h}$ / (DHA + (ATQ + TCS) $48 \mathrm{~h})$ compared to DHA (DHA $6 \mathrm{~h} /$ DHA $48 \mathrm{~h}$ ) means that the 1:1 combination ATQ + TCS is active on quiescent parasites. This can be correlated with the activity on quiescent parasites of ATQ alone with a difference of 12 days between (DHA $6 \mathrm{~h} /$ 
(DHA + ATQ) $48 \mathrm{~h}$ ) compared to DHA (DHA $6 \mathrm{~h} / \mathrm{DHA} 48 \mathrm{~h}$ ) and the maintained mitochondrial activity under the quiescence state. ${ }^{13,16,17}$ TCS alone didn't show any activity neither on proliferating parasites, nor on quiescent parasites with no delay observed between both first conditions. At the same dose tested $(7 \mu \mathrm{M})$, hybrid 4, compared to the 1:1 ATQ + TCS combination, showed a reduced activity in the quiescent parasites evidenced by a delay of only 3 days between conditions (DHA 6 h / (DHA + 4) 48 h) and DHA (DHA 6 h / DHA 48 h). This loss of activity of the hybrid $\mathbf{4}$ is also illustrated, on proliferating parasites, by 10 days necessary for parasites to reach initial parasitemia comparatively to 20 days when they are treated by the corresponding combination ATQ + TCS (Table 4, third column).

Table 4: QSA: Recrudescence time of the P. falciparum ART-resistant line F32-ART5

\begin{tabular}{cccc}
\hline \multirow{2}{*}{ Compounds } & \multicolumn{3}{c}{ Median recrudescence days } \\
\cline { 2 - 4 } & $\begin{array}{c}\text { DHA 6 h then } \\
\text { DHA 48 h }\end{array}$ & $\begin{array}{c}\text { DHA 6 h then } \\
\text { (DHA+Molecule) } 48 \text { h }\end{array}$ & $\begin{array}{c}\text { Nothing 6 h then } \\
\text { Molecule 48 h }\end{array}$ \\
\hline ATQ & 6 & 18 & 17 \\
\hline TCS & 7 & 7 & 20 \\
\hline ATQ + TCS $(1: 1)$ & 7 & 20 & 10 \\
\hline Hybrid 4 & 7 & 10 & $>30$ \\
\hline Chloroquine $(200 \mathrm{nM})^{\mathrm{a}}$ & 6 & 8 & \\
\hline
\end{tabular}

Synchronized ring-stage parasites have different drug treatment during $54 \mathrm{~h}$. After culture washing, the parasitemia was monitored until reaching the initial parasitemia, defined as the recrudescence day. Delay in recrudescence times corresponds to the difference between the recrudescence day after DHA $6 \mathrm{~h} /(\mathrm{DHA}+$ molecule) $48 \mathrm{~h}$ exposure and the recrudescence day after DHA alone $(54 \mathrm{~h})$ treatment. All the molecules were tested at $7 \mu \mathrm{M}$ (except chloroquine at $200 \mathrm{nM}$ ), in 3 independent experiments. The control condition (nothing $6 \mathrm{~h} /$ molecule 48 h) allowed the confirmation of the antiplasmodial activity of the molecules tested alone at this concentration. ATQ: atovaquone; TCS: triclosan.

a data from reference ${ }^{[17]}$

In conclusion, 1:1 combinations of three compounds having good antiplasmodial activities, atovaquone, mefloquine, GW844520, and of triclosan, an inhibitor of the lipid metabolism, were evaluated against $P$. falciparum parasites. Corresponding hybrid molecules combining atovaquone and triclosan, GW844520 and triclosan and, atovaquone and mefloquine were 
synthesized, focusing on rapid and efficient drugs linking, and studied in similar conditions to allow a first comparison between drug combination and drug hybridization. All combinations and hybrids showed high activity on proliferative $P$. falciparum parasites, with $\mathrm{IC}_{50}$ values in the low- or sub-nanomolar range for ATQ + TCS, GW844520 + TCS, ATQ + MQ and hybrids 4, 12 and 13, together with very good selectivity towards mammalian cells. Interestingly, in a context of artemisinin resistance, no cross-resistance with artemisinin was observed for these combinations and hybrids. Moreover, the combination ATQ + TCS showed a strong activity against parasites at the DHA-induced quiescence state. However, at high concentrations, the corresponding hybrid $\mathbf{4}$ showed a weaker activity than the combination on proliferative and on quiescent parasites, suggesting a lower solubility. This result does not call into question the value of using hybrid molecules to combat resistant parasites. Indeed, the combination of two molecules, each having different targets and presenting no cross-resistance with artemisinin, in a hybrid compound would be a good approach to prevent cross-resistance and kill quiescent parasites. That is why, based on the very promising results obtained with all combinations tested, further studies will be carried out on these hybrids focusing on linker pharmacomodulation. Thus, varying the nature of the linker with an oxygenated chain, modifying its length and the nature of the chemical bond for drug linkage (e.g. $O$-alkylation versus $O$-esterification) would certainly impact the solubility, the stability and more generally the activity of the resulting hybrids. Furthermore, the use of a combination active on quiescent parasites such as atovaquone combined with triclosan represents an encouraging prospect for the development of effective treatment of malaria cases caused by artemisinin-resistant parasites.

\section{Supplementary data}

Supplementary data to this article can be found online at .....

\section{Acknowledgments}

This study was supported in part by the French "Agence Nationale de la Recherche" (ANR grant INMAR ANR16 CE35 0003), the "Centre National de la Recherche Scientifique" and the Sanofi-Institut Pasteur award. 
Christian Bijani from the Laboratoire de Chimie de Coordination and Catherine Claparols, Nathalie Martins-Froment, Valérie Bourdon and Eric Leroy from the Mass spectrometry service of the ICT-FR 2599 (Toulouse) are acknowledged for their support, respectively for NMR and HR-MS experiments.

\section{References}

1. World Health Organisation. World Malaria Report.; 2020. https://www.who.int/publications/i/item/world-malaria-report-2019

2. World Health Organisation. Status Report on Artemisinin and ACT Resistance.; 2015. doi:10.1017/CBO9781107415324.004

3. Wongsrichanalai C, Meshnick SR. Declining artesunate-mefloquine efficacy against falciparum malaria on the Cambodia-Thailand border. Emerg Infect Dis. 2008;14(5):716-719. doi:10.3201/eid1405.071601

4. Noedl H, Se Y, Schaecher K, Smith BL, Socheat D, Fukuda MM. Evidence of Artemisinin-resistant malaria in Western Cambodia. N Engl J Med. 2008;359:26192620. doi:10.1056/NEJMc0805011

5. Ashley EA, Dhorda M, Fairhurst RM, et al. Spread of Artemisinin resistance in Plasmodium falciparum malaria. $N$ Engl $J$ Med. 2014;371:411-423. doi:10.1056/NEJMoa1314981

6. Dondorp AM, Nosten F, Yi P, et al. Artemisinin resistance in Plasmodium falciparum malaria. N Engl J Med. 2009;361(5):455-467. doi:10.1056/NEJMoa0808859

7. Ménard D, Khim N, Beghain J, et al. A worldwide map of Plasmodium falciparum K13propeller polymorphisms. $N$ Engl J Med. 2016;374(25):2453-2464. doi:10.1056/NEJMoa1513137

8. Amaratunga C, Lim P, Suon S, et al. Dihydroartemisinin-piperaquine resistance in Plasmodium falciparum malaria in Cambodia: a multisite prospective cohort study. Lancet Infect Dis. 2016;16(3):357-365. doi:https://doi.org/10.1016/S14733099(15)00487-9

9. Duru V, Khim N, Leang R, Kim S, Domergue A, Kloeung N. Plasmodium falciparum 
dihydroartemisinin-piperaquine failures in Cambodia are associated with mutant K13 parasites presenting high survival rates in novel piperaquine in vitro assays: retrospective and prospective investigations. BMC Med. 2015;13:305. doi:10.1186/s12916-015-05395

10. Witkowski B, Lelievre J, Barragan MJL, et al. Increased tolerance to artemisinin in Plasmodium falciparum is mediated by a quiescence mechanism. Antimicrob Agents Chemother. 2010;54(5):1872-1877. doi:10.1128/AAC.01636-09

11. Ariey F, Witkowski B, Amaratunga C, et al. A molecular marker of artemisinin-resistant Plasmodium falciparum malaria. Nature. 2014;505(7481):50-55. doi:10.1038/nature12876

12. Teuscher F, Gatton ML, Chen N, Peters J, Kyle DE, Cheng Q. Artemisinin-induced dormancy in Plasmodium falciparum: duration, recovery rates, and implications in treatment failure. J Infect Dis. 2010;202(9):1362-1368. doi:10.1086/656476

13. Chen N, LaCrue AN, Teuscher F, Waters NC, Gatton ML, Kyle DE. Fatty acid synthesis and pyruvate metabolism pathways remain active in dihydroartemisinin-induced dormant ring stages of Plasmodium falciparum. Antimicrob Agents Chemother. 2014;58(8):4773-4781. doi:10.1128/AAC.02647-14

14. Paloque L, Ramadani AP, Mercereau-Puijalon O, Augereau J-M, Benoit-Vical F. Plasmodium falciparum: multifaceted resistance to artemisinins. Malar J. 2016;15(15):149. doi:10.1186/s12936-016-1206-9

15. Nixon GL, Pidathala C, Shone AE, et al. Targeting the mitochondrial electron transport chain of Plasmodium falciparum: New strategies towards the development of improved antimalarials for the elimination era. Future Med Chem. 2013;5(13):1573-1591. doi:10.4155/fmc.13.121

16. Peatey CL, Chavchich M, Chen N, Gresty KJ, Gray KA, Gatton ML. Mitochondrial membrane potential in a small subset of Artemisinin-induced dormant Plasmodium falciparum parasites in vitro. J Infect Dis. 2015;212:426-434. doi:10.1093/infdis/jiv048

17. Reyser T, Paloque L, Ouji M, et al. Identification of compounds active against quiescent artemisinin-resistant Plasmodium falciparum parasites via the quiescent stage survival assay (QSA). J Antimicrob Chemother. 2020;75(10):2826-2834. doi:https://doi.org/10.1093/jac/dkaa250 
18. Capper MJ, O’Neill PM, Fisher N, et al. Antimalarial 4(1H)-pyridones bind to the Qi site of cytochrome bc1. Proc Natl Acad Sci. 2015;112(3):755 LP - 760. doi:10.1073/pnas.1416611112

19. Menard S, Ben Haddou T, Ramadani AP, et al. Induction of multidrug tolerance in Plasmodium falciparum by extended artemisinin pressure. Emerg Infect Dis. 2015;21(10):1733-1741. doi:10.3201/eid2110.150682

20. Morphy R, Rankovic Z. Designed multiple ligands. An emerging drug discovery paradigm. J Med Chem. 2005;48(21):6523-6543. doi:10.1021/jm058225d

21. Morphy R, Kay C, Rankovic Z. From magic bullets to designed multiple ligands. Drug Discov Today. 2004;9(15):641-651. doi:https://doi.org/10.1016/S1359-6446(04)031630

22. Agarwal D, Gupta RD, Awasthi SK. Are antimalarial hybrid molecules a close reality or a distant dream? Antimicrob Agents Chemother. 2017;61(5):e00249-17. doi:10.1128/AAC.00249-17

23. Feng $\mathrm{L}, \mathrm{Xu} \mathrm{Z}$, Chang $\mathrm{L}$, et al. Hybrid molecules with potential in vitro antiplasmodial and in vivo antimalarial activity against drug-resistant Plasmodium falciparum. Med Res Rev. Published online 2019:1-41. doi:10.1002/med.21643

24. Fry M, Pudney M. Site of action of the antimalarial hydroxynaphthoquinone, 2-[trans-4(4'-chlorophenyl) cyclohexyl]-3- hydroxy-1,4-naphthoquinone (566C80). Biochem Pharmacol. 1992;43(7):1545-1553. doi:https://doi.org/10.1016/0006-2952(92)90213-3

25. Perozzo R, Kuo M, Sidhu A bir S, et al. Structural elucidation of the specificity of the antibacterial agent triclosan for malarial enoyl acyl carrier protein reductase. J Biol Chem. 2002;277(15):13106-13114. doi:10.1074/jbc.M112000200

26. Surolia N, Surolia A. Triclosan offers protection against blood stages of malaria by inhibiting enoyl-ACP reductase of Plasmodium falciparum. Nat Med. 2001;7(2):167173. doi:10.1038/84612

27. Zhang H, Tweel B, Tong L. Molecular basis for the inhibition of the carboxyltransferase domain of acetyl-coenzyme-A carboxylase by haloxyfop and diclofop. Proc Natl Acad Sci U S A. 2004;101(16):5910 LP - 5915. doi:10.1073/pnas.0400891101

28. Capper MJ, O’Neill PM, Fisher N, et al. Antimalarial 4(1H)-pyridones bind to the Qi 
site of cytochrome bc1. Proc Natl Acad Sci. 2015;112(3):755-760. doi:10.1073/pnas.1416611112

29. Gunjan S, Singh SK, Sharma T, et al. Mefloquine induces ROS mediated programmed cell death in malaria parasite: Plasmodium. Apoptosis. 2016;21(9):955-964. doi:10.1007/s10495-016-1265-y

30. Chevli R, Fitch CD. The antimalarial drug mefloquine binds to membrane phospholipids. Antimicrob Agents Chemother. 1982;21(4):581-586. doi:10.1128/aac.21.4.581

31. Wong W, Bai X-C, Sleebs BE, et al. Mefloquine targets the Plasmodium falciparum 80S ribosome to inhibit protein synthesis. Nat Microbiol. 2017;2(6):17031. doi:10.1038/nmicrobiol.2017.31

32. Witkowski B, Amaratunga C, Khim N, et al. Novel phenotypic assays for the detection of artemisinin-resistant Plasmodium falciparum malaria in Cambodia: In-vitro and exvivo drug-response studies. Lancet Infect Dis. 2013;13(12):1043-1049. doi:10.1016/S1473-3099(13)70252-4

33. El Hage S, Ane M, Stigliani J-L, et al. Synthesis and antimalarial activity of new atovaquone derivatives. Eur J Med Chem. 2009;44(11):4778-4782. doi:https://doi.org/10.1016/j.ejmech.2009.07.021

34. Mishra S, Karmodiya K, Parasuraman P, Surolia A, Surolia N. Design, synthesis, and application of novel triclosan prodrugs as potential antimalarial and antibacterial agents. Bioorg Med Chem. 2008;16(10):5536-5546. doi:https://doi.org/10.1016/j.bmc.2008.04.006

35. Comley JC, Yeates CL, Frend TJ. Antipneumocystis activity of 17C91, a prodrug of atovaquone. Antimicrob Agents Chemother. 1995;39(10):2217-2219. http://www.ncbi.nlm.nih.gov/pmc/articles/PMC162917/

36. Romeo S, Parapini S, Dell'Agli M, et al. Atovaquone-Statine "Double-Drugs" with High Antiplasmodial Activity. ChemMedChem. 2008;3(3):418-420. doi:10.1002/cmdc.200700166

37. Yeates CL, Batchelor JF, Capon EC, et al. Synthesis and structure-activity relationships of 4-pyridones as potential antimalarials. J Med Chem. 2008;51(9):2845-2852. doi:10.1021/jm0705760 
38. Bueno JM, Calderon F, Chicharro J, et al. Synthesis and structure-activity relationships of the novel antimalarials 5-pyridinyl-4(1H)-pyridones. J Med Chem. 2018;61(8):34223435. doi:10.1021/acs.jmedchem.7b01256

39. Grellepois F, Grellier P, Bonnet-Delpon D, Bégué J-P. Design, synthesis and antimalarial activity of trifluoromethylartemisinin-mefloquine dual molecules. ChemBioChem. 2005;6(4):648-652. doi:10.1002/cbic.200400347

40. Bueno JM, Manzano P, García MC, et al. Potent antimalarial 4-pyridones with improved physico-chemical properties. Bioorg Med Chem Lett. 2011;21(18):5214-5218. doi:https://doi.org/10.1016/j.bmcl.2011.07.044 